\title{
A Tight Closed-Form Approximation of the Log-Normal Fading Channel Capacity
}

\author{
Fabien Héliot, Member, IEEE, Xiaoli Chu, Member, IEEE, Reza Hoshyar, Member, IEEE, \\ and Rahim Tafazolli, Member, IEEE
}

\begin{abstract}
The log-normal probability distribution has been commonly used in wireless communications to model the shadowing and, recently, the small-scale fading for indoor ultrawideband (UWB) communications. In this paper, a tight closedform approximation of the ergodic capacity over log-normal fading channels is derived. This expression can be easily used to evaluate and compare the ergodic capacities of communication systems operating over log-normal fading channels. We also utilize this expression to show that the capacity of a multi-antennas UWB system operating over the IEEE 802.15.3a channel can be improved mainly through receive diversity.
\end{abstract}

Index Terms-Log-normal distribution, fading channels, ergodic capacity, closed-form, hyperbolic functions.

\section{INTRODUCTION}

$\mathbf{T}$ HE log-normal distribution has long been used predominantly in communications to model the effect of shadowing due to large obstructions [1], i.e., medium-scale fading. Recently, the log-normal distribution has been applied to describe the small-scale fading in the IEEE 802.15.3a channel model [2], for indoor ultra-wideband (UWB) communications [3]. More generally, the amplitude distribution of slowly varying communication channels, e.g., indoor environments, tends to be log-normal [4].

Channel capacity and probability of error are common metrics used to evaluate the performance of communication systems. The calculations of channel capacity and probability of error over typical fading channels, such as Rayleigh, Rician, Nakagami, and Hoyt, have attracted considerable research interests over the past decade [5]-[11]. The error probability of communication systems operating over log-normal fading channels was commonly expressed using a computable formula in [8] until an accurate closed-form approximation was derived in [12]. As for the capacity, it was first upper and lower bounded in [13]. These two bounds are closed-form expressions but unfortunately are loose for low signal-to-noise ratio (SNR) values. Recently in [4], an accurate computable formula, which is the truncation of an infinite alternating series and is not a closed-form expression, was proposed for evaluating the capacity of log-normal fading channels.

Manuscript received July 22, 2008; revised October 3, 2008, January 21, 2009, and March 18, 2009; accepted March 19, 2009. The associate editor coordinating the review of this letter and approving it for publication was C.-X. Wang.

F. Héliot, R. Hoshyar, and R. Tafazolli are with the Centre for Communication Systems Research, University of Surrey, Faculty of Electronics \& Physical Sciences, Guildford GU2 7XH, UK (e-mail: F.Heliot@Surrey.ac.uk).

$\mathrm{X}$. Chu is with the UWB Communications Group, Division of Engineering, King's College London, University of London, London WC2R 2LS, UK.

Digital Object Identifier 10.1109/TWC.2009.080972
This formula was refined in [14] and a new closed-form approximation of the capacity was also derived in [14].

In this paper, we propose a tight closed-form approximation of the ergodic capacity over log-normal fading channels, relying on the system model introduced in Section II. In Section III, we first derive a very tight closed-form approximation of the conventional integral formulation of the ergodic capacity [13] for low dB spread values; we then show that this expression is the greatest lower bound (GLB) on the ergodic capacity in terms of $\mathrm{dB}$ spread for any SNR values. In Section IV, we design a parametric function that best fits the difference between the exact ergodic capacity and its GLB, by using a heuristic curve fitting method [9][12], [15]. We obtain a tight closed-form approximation of the ergodic capacity, which is a function of the system SNR and the dB spread of the log-normal fading channel, by minimizing this difference. Numerical results show that our closed-form approximation differs from the conventional integral formulation of the ergodic capacity [13] by less than 0.01 nats $/ \mathrm{s} / \mathrm{Hz}$ for any SNR and $\mathrm{dB}$ spread values lying in the range of interest for wireless communications. Our expression is more simplified than the computable formula proposed in [4] and [14], but with similar accuracy, and is far more accurate than the closed-form approximation derived in [14], as it is evidently shown in Section IV. In Section V, we use our expression to interpret the behavior of the capacity at high SNRs and we provide a criterion to easily compare the capacities of any two systems operating over log-normal fading channels. We also utilize this expression to compute the ergodic capacities of single-input single-output (SISO) and multiple-input multiple-output (MIMO) UWB systems operating over the IEEE 802.15.3a channel, and show that multi-antennas UWB systems can mainly benefit from receive diversity to increase their capacities. Finally, conclusions are drawn in Section VI. Some preliminary results of this work have been presented in [16].

\section{SySTEM MODEL FOR LOG-NORMAL FADING CHANNEL CAPACITY}

In wireless communications, medium-scale fading is usually modeled as a multiplicative and slowly time-varying random process. Accordingly, the received signal $r(t)$ is given by [1]

$$
r(t)=\sqrt{\epsilon} s(t) h(t)+n(t),
$$

where $\epsilon$ is the average transmitted signal energy, $s(t)$ is the transmitted signal, $h(t)$ is the random process that characterizes medium-scale fading, and $n(t)$ is an additive white Gaussian noise (AWGN) process with a double-sided variance 
of $N_{0} / 2$. Medium-scale fading is considered as log-normally distributed for any values of $t$. In a slow-varying environment, $h(t)$ can be considered constant over multiple symbol periods such that $h(t)=\beta$ is a log-normally distributed random variable $(\mathrm{RV})$. As far as indoor UWB communication is concerned, the multipath attenuation factors of the IEEE 802.15.3a channel model [2] are log-normally distributed, and the received signal $r(t)$ can be expressed as in (1) with $h(t)=\sum_{k} G_{k} \delta\left(t-\tau_{k}\right)$ [17], [18], where $\delta(t)$ denotes the Dirac delta function, $\tau_{k}$ is the $k$-th time delay, $G_{k}=\beta_{k} e^{j \theta_{k}}$ is the $k$-th multipath attenuation factor, $\beta_{k}$ is a log-normal RV, and $\theta_{k} \in\{0, \pi\}$ is an uniformly distributed RV [2].

The ergodic capacity of a log-normal fading channel is expressed as follows [13]

$$
C_{\mathrm{e}}=\mathrm{E}[\ln (1+\gamma \alpha)]=2 \lambda \int_{0}^{+\infty} \ln (1+\gamma \alpha) p(\alpha) d \alpha
$$

where $\gamma=\epsilon / N_{0}$ is the average SNR, $\lambda=\frac{1}{2}$ if the capacity is expressed in nats/s/Hz or $\lambda=\frac{1}{2 \ln (2)}$ if the capacity is expressed in bits $/ \mathrm{s} / \mathrm{Hz}$, and $p(\alpha)=$ $\frac{1}{\alpha \sqrt{2 \pi} \sigma_{\alpha}} \exp \left(-\frac{1}{2}\left[\left(\ln (\alpha)-m_{\alpha}\right) / \sigma_{\alpha}\right]^{2}\right)$ is the probability density function of a log-normally distributed $\mathrm{RV} \alpha$, where $m_{\alpha}$ and $\sigma_{\alpha}$ are the mean and standard deviation of $\ln (\alpha)$, respectively. Concerning medium-scale fading, $\alpha=\beta^{2}$, where $\beta^{2}$ is log-normally distributed since $\beta$ is log-normally distributed too. In the case of indoor UWB channel, $\alpha \simeq \sum_{k} \beta_{k}^{2}$, as shown in [17] and [18]. According to the results in [19]-[21] and the novel method recently proposed in [22], the sum of log-normal RVs can be well-approximated by an equivalent log-normal RV for a wide range of $\mathrm{dB}$ spread values. Thus, the ergodic capacity formula in (2) can be applied to both medium-scale fading and indoor UWB channel.

In wireless communications, parameters $\left(m_{y}, \sigma_{y}\right)$ are introduced to characterize a log-normal distribution, where $m_{y}=\kappa m_{\alpha}, \sigma_{y}=\kappa \sigma_{\alpha}$ and $\kappa=10 / \ln (10)$ [19]. The parameter $\sigma_{y}$, known as the $\mathrm{dB}$ spread, is between 6 and 12 $\mathrm{dB}$ for medium-scale fading [19], between 3 and $5 \mathrm{~dB}$ for the SISO indoor UWB channel [2], and between 1 and $4 \mathrm{~dB}$ for the MIMO indoor UWB channel as computed in Tables 5.1 and 5.2 of [23]. Without loss of generality, its range of values can be aggregated as $\sigma_{y} \in[1,12] \mathrm{dB}$ for most communication systems operating over log-normal fading channels. Applying the change of variables $x=\frac{\ln (\alpha)-m_{\alpha}}{\sqrt{2} \sigma_{\alpha}}$ in $p(\alpha), C_{\mathrm{e}}$ in (2) is rewritten as

$$
C_{\mathrm{e}}=\lambda \int_{-\infty}^{+\infty} \ln \left(1+e^{\left(\frac{1}{\kappa}\left(\sqrt{2} \sigma_{y} x+m_{y}+\gamma_{\mathrm{dB}}\right)\right)}\right) \frac{2}{\sqrt{\pi}} e^{-x^{2}} d x,
$$

where $\gamma_{\mathrm{dB}}=\kappa \ln (\gamma)$. Notice in (3) that $m_{y}$ offsets the SNR value $\gamma_{\mathrm{dB}}$ and these two terms can be aggregated as $\widehat{\gamma}_{\mathrm{dB}}=$ $\gamma_{\mathrm{dB}}+m_{y}$. The expression in (3) can then be re-expressed by using the hyperbolic function $\cosh (u)=\left(e^{u}+e^{-u}\right) / 2$, as follows,

$$
\begin{aligned}
C_{\mathrm{e}} & =\lambda \int_{-\infty}^{+\infty}\left[\ln (2)+\frac{\widehat{\gamma}_{\mathrm{dB}}}{2 \kappa}+\ln \left(\cosh \left(\frac{\sqrt{2} \sigma_{y} x+\widehat{\gamma}_{\mathrm{dB}}}{2 \kappa}\right)\right)\right. \\
& \left.+\frac{\sqrt{2} \sigma_{y} x}{2 \kappa}\right] \frac{2}{\sqrt{\pi}} e^{-x^{2}} d x .
\end{aligned}
$$

Also, knowing that $\int_{-\infty}^{+\infty} x e^{-x^{2}} d x=0$ and $\int_{0}^{+\infty} e^{-x^{2}} d x=$ $\sqrt{\pi} / 2$, (4) can be simplified as

$$
C_{\mathrm{e}}=\lambda\left[2 \ln (2)+\frac{\widehat{\gamma}_{\mathrm{dB}}}{\kappa}+\int_{-\infty}^{+\infty} f(x) g(x) d x\right],
$$

where $f(x)=\ln \left(\cosh \left(\frac{\sqrt{2} \sigma_{y} x+\widehat{\gamma}_{\mathrm{dB}}}{2 \kappa}\right)\right)$, and $g(x)=\frac{2}{\sqrt{\pi}} e^{-x^{2}}$.

\section{Approximating the Integral of $f(x) g(x)$ IN (5)}

Using the integration by parts defined as $\int_{a}^{b} f(x) g(x) d x=[f(x) G(x)]_{a}^{b}-\int_{a}^{b} \frac{d f(x)}{d x} G(x) d x$, with $\frac{d f(x)}{d x}=\frac{\sigma_{y}}{\sqrt{2} \kappa} \tanh \left(\frac{\sqrt{2} \sigma_{y} x+\widehat{\gamma}_{\mathrm{dB}}}{2 \kappa}\right)$ and $G(x)=\operatorname{erf}(x)$, and since $\int_{-\infty}^{+\infty} f(x) g(x) d x=\lim _{s \rightarrow+\infty} \int_{-s}^{s} f(x) g(x) d x=$ $\lim _{s \rightarrow+\infty} \int_{0}^{s}[f(-x)+f(x)] g(x) d x$, the ergodic capacity in (5) can be re-expressed as

$$
\begin{aligned}
C_{\mathrm{e}} & =\lim _{s \rightarrow+\infty} \lambda\left\{2 \ln (2)+\frac{\widehat{\gamma}_{\mathrm{dB}}}{\kappa}+[(f(-x)+f(x)) G(x)]_{0}^{s}\right. \\
& \left.-\int_{0}^{s}\left(\frac{d f(-x)}{d x}+\frac{d f(x)}{d x}\right) G(x) d x\right\} .
\end{aligned}
$$

Knowing that $G(0)=0, \lim _{s \rightarrow+\infty} G(s)=1$, and

$$
\begin{aligned}
\lim _{s \rightarrow+\infty} & (f(-s)+f(s))=-2 \ln (2)+\frac{2 \sigma_{y}}{\sqrt{2} \kappa} \lim _{s \rightarrow+\infty} \int_{0}^{s} d_{x}, \\
C_{\mathrm{e}} & =\lim _{s \rightarrow+\infty} \lambda\left\{\frac{\widehat{\gamma}_{\mathrm{dB}}}{\kappa}+\frac{\sigma_{y}}{\sqrt{2} \kappa}\left[\int_{0}^{s} 2 d_{x}+\int_{0}^{s} \operatorname{erf}(x)\right.\right. \\
& \left.\left.\times \sum_{i=0}^{1}(-1)^{i} \tanh \left(\frac{(-1)^{i+1} \sqrt{2} \sigma_{y} x+\widehat{\gamma}_{\mathrm{dB}}}{2 \kappa}\right) d x\right]\right\}, \\
= & \frac{\lambda \widehat{\gamma}_{\mathrm{dB}}}{\kappa}+\frac{\lambda \sigma_{y}}{\sqrt{2} \kappa} \lim _{s \rightarrow+\infty} \int_{0}^{s} h(x) d x \\
= & \frac{\lambda \widehat{\gamma}_{\mathrm{dB}}}{\kappa}+\frac{\lambda \sigma_{y}}{\sqrt{2} \kappa} \int_{0}^{+\infty} h(x) d x,
\end{aligned}
$$

where $h(x)=\left(\tanh \left(\frac{-\sqrt{2} \sigma_{y} x+\widehat{\gamma}_{\mathrm{dB}}}{2 \kappa}\right)-\tanh \left(\frac{\sqrt{2} \sigma_{y} x+\widehat{\gamma}_{\mathrm{dB}}}{2 \kappa}\right)\right)$ $\times \operatorname{erf}(x)+2$, and $\operatorname{erf}(x)$ is the error function. Over the interval $[0,+\infty)$, the function $\operatorname{erf}(x)$ can be well-approximated by $\tanh (a x)$, with $a \in[1.13,1.32]$. The functions $\operatorname{erf}(x)$ and $\tanh (a x)$ differ on average by less than 5\% for this range of $a$ values. Substituting $\operatorname{erf}(x)$ by $\tanh (a x)$ in $h(x)$ and knowing that $\tanh (x)=1-2 /[\exp (2 x)+1]=-1+2 /[\exp (-2 x)+1]$, $C_{\mathrm{e}}$ in (7) can be approximated as

$$
\begin{aligned}
C_{\mathrm{e}} & \simeq \frac{\lambda \widehat{\gamma}_{\mathrm{dB}}}{\kappa}+\frac{\lambda \sqrt{2} \sigma_{y}}{\kappa} \int_{0}^{+\infty}\left[\left(1-\frac{2}{e^{2 a x}+1}\right)\right. \\
& \left.\times\left(-1+\sum_{i=0}^{1} \frac{1}{e^{\frac{1}{\kappa}\left(\sqrt{2} \sigma_{y} x+(-1)^{i} \widehat{\gamma}_{\mathrm{dB}}\right)}+1}\right)+1\right] d x .
\end{aligned}
$$

Applying the change of variable $u=e^{x}$ in (8) and a number of simplifications, we obtain

$$
\begin{aligned}
C_{\mathrm{e}} & \simeq 2 \lambda\left[\left(1+\frac{\sigma_{y}}{\sqrt{2} a \kappa}\right) \ln (2)+\frac{\widehat{\gamma}_{\mathrm{dB}}}{2 \kappa}+\ln \left(\cosh \left(\frac{\widehat{\gamma}_{\mathrm{dB}}}{2 \kappa}\right)\right)\right] \\
& -\frac{2 \sqrt{2} \lambda \sigma_{y}}{\kappa} \int_{1}^{+\infty}\left(\frac{d u}{q\left(a, \sigma_{y}, \widehat{\gamma}_{\mathrm{dB}}, u\right)}+\frac{d u}{q\left(a, \sigma_{y},-\widehat{\gamma}_{\mathrm{dB}}, u\right)}\right),
\end{aligned}
$$




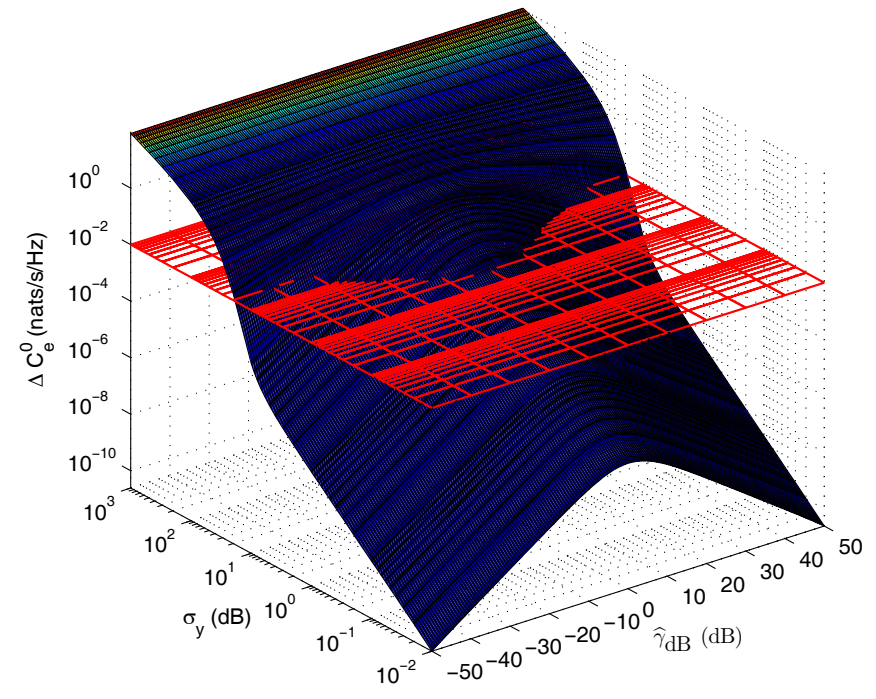

Fig. 1. Approximation error of $C_{\mathrm{e}}^{0}$ against $C_{\mathrm{e}}$ in nats/s/Hz.

where $q\left(a, \sigma_{y}, \widehat{\gamma}_{\mathrm{dB}}, u\right)=e^{\frac{\hat{\gamma}_{\mathrm{dB}}}{\kappa}} u^{\frac{2 a \kappa+\sqrt{2} \sigma_{y}}{\kappa}+1}+u^{2 a+1}+$ $e^{\frac{\hat{\gamma}_{\mathrm{BB}}}{\kappa}} u^{\frac{\sqrt{2} \sigma_{y}}{\kappa}+1}+u$ is a polynomial. The integral part in (9) can then be solved by expanding the rational functions $1 / q\left(a, \sigma_{y}, \pm \widehat{\gamma}_{\mathrm{dB}}, u\right)$ into partial fractions. As long as the roots of $q\left(a, \sigma_{y}, \pm \widehat{\gamma}_{\mathrm{dB}}, u\right)$ are known, the expression in (9) will be a generic and accurate closed-form approximation of the ergodic capacity.

In the case of low $\sigma_{y}$ values, i.e., $\sigma_{y} \ll \kappa / \sqrt{2}$, $q\left(a, \sigma_{y}, \widehat{\gamma}_{\mathrm{dB}}, u\right) \simeq\left(e^{\frac{\hat{\gamma}_{\mathrm{dB}}}{\kappa}}+1\right) u\left(u^{2 a}+1\right), \quad$ and $\int_{1}^{+\infty} \frac{1}{q\left(a, \hat{\gamma}_{\mathrm{dB}}, u\right)} d u=\ln (2) /\left[2 a\left(e^{\frac{\hat{\gamma}_{\mathrm{dB}}}{\kappa}}+1\right)\right]$. Using this result in (9) and applying some simplifications, a closed-form approximation of $C_{\mathrm{e}}$ for low $\sigma_{y}$ values is given by

$$
C_{\mathrm{e}} \simeq C_{\mathrm{e}}^{0}=2 \lambda\left[\frac{\widehat{\gamma}_{\mathrm{dB}}}{2 \kappa}+\ln \left(2 \cosh \left(\frac{\widehat{\gamma}_{\mathrm{dB}}}{2 \kappa}\right)\right)\right] .
$$

The accuracy of our proposed approximation $C_{\mathrm{e}}^{0}$ is measured, as in [14], by using the metric $\Delta C_{\mathrm{e}}^{0}=\left|C_{\mathrm{e}}-C_{\mathrm{e}}^{0}\right|$, where $\Delta C_{\mathrm{e}}^{0}$ represents the approximation error between $C_{\mathrm{e}}^{0}$ and $C_{\mathrm{e}}$ in nats/s/Hz. As depicted in Fig. 1, where $\Delta C_{\mathrm{e}}^{0}$ is plotted against $\sigma_{y}$ and $\widehat{\gamma}_{\mathrm{dB}}, C_{\mathrm{e}}^{0}$ is accurate for low $\sigma_{y}$ values, i.e., $\sigma_{y} \leq 2 \mathrm{~dB}$, regardless of $\widehat{\gamma}_{\mathrm{dB}}$, and for $\sigma_{y} \in[0.01,20] \mathrm{dB}$ with high $\widehat{\gamma}_{\mathrm{dB}}$ values, i.e., $\widehat{\gamma}_{\mathrm{dB}} \geq 30 \mathrm{~dB}$. The accuracy of $C_{\mathrm{e}}^{0}$ increases as $\sigma_{y}$ gets lower. Moreover, for any $x \in \mathbb{R}, \sigma_{y} \geq 0$ and $\widehat{\gamma}_{\mathrm{dB}} \in \mathbb{R}$, the inequalities $f(x) \geq f(0) \geq 0$ and $g(x) \geq 0$ hold. Therefore, $f(x) g(x) \geq f(0) g(x), \int_{-\infty}^{+\infty} f(x) g(x) d x \geq \int_{-\infty}^{+\infty} f(0) g(x) d x$, equivalently $C_{\mathrm{e}} \geq \lambda\left[2 \ln (2)+\frac{\widehat{\gamma}_{\mathrm{dB}}}{\kappa}+\int_{-\infty}^{+\infty} f(0) g(x) d x\right]=$ $C_{\mathrm{e}}^{0}$, and then it implies that $C_{\mathrm{e}}^{0}$ is a lower bound of $C_{\mathrm{e}}$. Furthermore, $C_{\mathrm{e}}=C_{\mathrm{e}}^{0}$ for $\sigma_{y}=0$, thus, $C_{\mathrm{e}}^{0}=\inf \left\{\sigma_{y} \geq 0: C_{\mathrm{e}}\right\}$, i.e., $C_{\mathrm{e}}^{0}$ is the GLB of the ergodic capacity over $\log$-normal fading channels in terms of $\mathrm{dB}$ spread for any $\widehat{\gamma}_{\mathrm{dB}}$ values.

\section{A Generic Closed-Form Approximation of the ERGODIC CAPACITY}

The closed-form expression in (10) is a tight approximation of $C_{\mathrm{e}}$ regardless of $\widehat{\gamma}_{\mathrm{dB}}$ for $\sigma_{y} \in[0,2] \mathrm{dB}$, as depicted in Fig. 1, but is less accurate for higher practical values of dB spread. Using (10), here we derive a generic closed-form

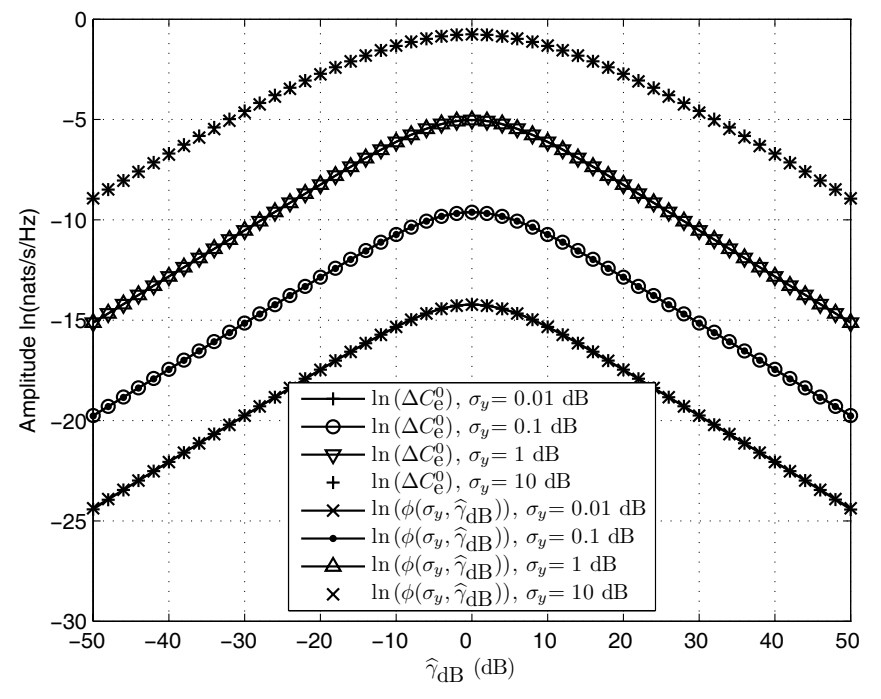

Fig. 2. Comparison of $\ln \left(\Delta C_{\mathrm{e}}^{0}\right)$ and $\ln \left(\phi\left(\sigma_{y}, \widehat{\gamma}_{\mathrm{dB}}\right)\right)$ for various $\sigma_{y}$ values.

approximation of the ergodic capacity which is tight regardless of $\widehat{\gamma}_{\mathrm{dB}}$ for $\mathrm{dB}$ spread values lying in the range of $[0.01,12]$ $\mathrm{dB}$.

Knowing that $C_{\mathrm{e}}^{0}$ is the GLB of $C_{\mathrm{e}}$, we aim to obtain a function $\phi\left(\sigma_{y}, \widehat{\gamma}_{\mathrm{dB}}\right)$ that tightly estimates the difference between $C_{\mathrm{e}}$ and $C_{\mathrm{e}}^{0}$, i.e., $\Delta C_{\mathrm{e}}^{0} \simeq \phi\left(\sigma_{y}, \widehat{\gamma}_{\mathrm{dB}}\right)$. In the heuristic curve fitting method proposed in [15], a parametric function is designed in terms of elementary functions and three independent parameters to solve a curve fitting problem. In this paper, we use this curve fitting method to design the parametric function $\phi\left(\sigma_{y}, \widehat{\gamma}_{\mathrm{dB}}\right)$ that tightly fits $\Delta C_{\mathrm{e}}^{0}$ for $\sigma_{y} \in[0.01,12]$ $\mathrm{dB}$ and a large range of $\widehat{\gamma}_{\mathrm{dB}}$ values. We first numerically evaluated $\ln \left(\Delta C_{\mathrm{e}}^{0}\right)$ for different values of $\sigma_{y}$ and collected the obtained curves in Fig. 2. It can be noticed that $\ln \left(\Delta C_{\mathrm{e}}^{0}\right)$ presents the feature of an even function with a unique maximum occurring at $\widehat{\gamma}_{\mathrm{dB}}=0 \mathrm{~dB}$, denoted by $\ln \left(\Delta C_{\mathrm{e}}^{0}(0)\right)$. In addition, this function decreases linearly as $\left|\widehat{\gamma}_{\mathrm{dB}}\right|$ increases. In the effort to obtain the function that best fits these curves, the curve fitting method leads to the parametric function $\ln \left(\phi\left(\sigma_{y}, \widehat{\gamma}_{\mathrm{dB}}\right)\right)=\ln \left(\Delta C_{\mathrm{e}}^{0}(0)\right)-\eta_{1} \ln \left(\cosh \left(\frac{\widehat{\gamma}_{\mathrm{dB}}}{\eta_{2}}\right)\right)$, which provides a satisfying approximation for any of the $\ln \left(\Delta C_{\mathrm{e}}^{0}\right)$ curves, as illustrated in Fig. 2, for $\sigma_{y}=0.01,0.1,1,10$ $\mathrm{dB}, \eta_{1}=2.01,2.01,2.02,4.2$, and $\eta_{2}=8.71,8.71,8.79,19$, respectively. The parameters $\ln \left(\Delta C_{\mathrm{e}}^{0}(0)\right), \eta_{1}$ and $\eta_{2}$ clearly depend on the value of $\sigma_{y}$, and their values increase as $\sigma_{y}$ increases, as illustrated in Figs. 1 and 2.

Consequently, we obtain that

$$
\phi\left(\sigma_{y}, \widehat{\gamma}_{\mathrm{dB}}\right)=\frac{\eta_{0}}{2 \kappa} e^{-\eta_{1} \ln \left(\cosh \left(\frac{\widehat{\gamma}_{\mathrm{dB}}}{2 \kappa \eta_{2}}\right)\right)}
$$

is an appropriate choice of a parametric function, with $\eta_{0}, \eta_{1}, \eta_{2} \geq 0$, in order to tightly approximate $\Delta C_{\mathrm{e}}^{0}$. The tightness of $\phi\left(\sigma_{y}, \widehat{\gamma}_{\mathrm{dB}}\right)$ can be controlled by adjusting the parameters $\eta_{0}, \eta_{1}$ and $\eta_{2}$. We minimize the following mean squared error (MSE) equation for tightly approximating $C_{\mathrm{e}}$ in (5) by $C_{\mathrm{e}}^{0}+\phi\left(\sigma_{y}, \widehat{\gamma}_{\mathrm{dB}}\right)$

$$
\frac{1}{2 N+1} \sum_{\gamma_{\mathrm{dB}}=-N}^{N}\left|\Delta C_{\mathrm{e}}^{0}-\phi\left(\sigma_{y}, \widehat{\gamma}_{\mathrm{dB}}\right)\right|^{2} \leq \varepsilon_{0},
$$




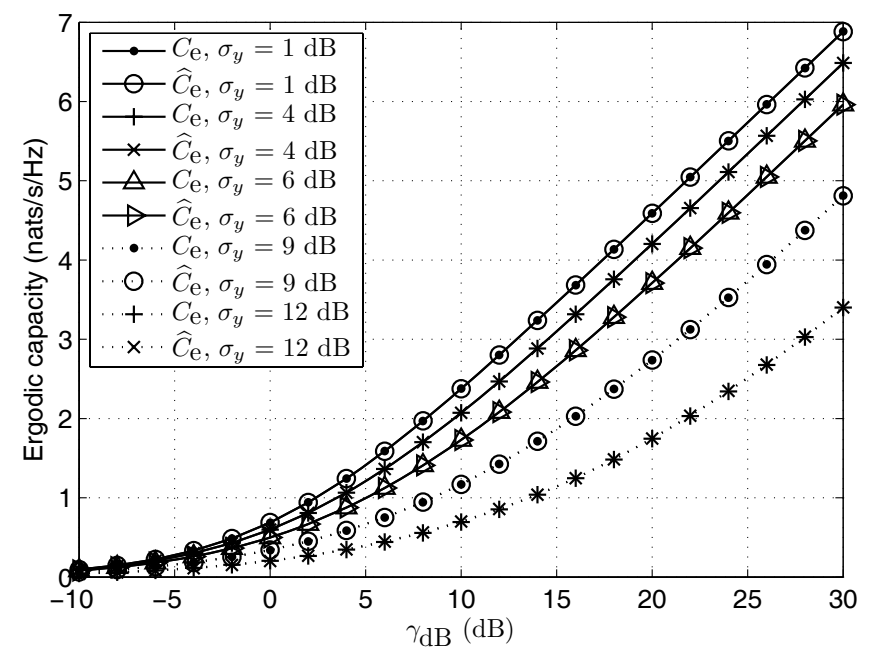

Fig. 3. Comparison of $C_{\mathrm{e}}$, and $\widehat{C}_{\mathrm{e}}$ vs. $\gamma_{\mathrm{dB}}$, for various $\sigma_{y}$ values, and $m_{y}=-\sigma_{y}^{2} / 2 \kappa$.

where $\varepsilon_{0} \ll 1$. Using (12), we have obtained $\eta_{0}, \eta_{1}$, and $\eta_{2}$, for $\sigma_{y} \in[0.01,12] \mathrm{dB}$ with an increment step of $0.1 \mathrm{~dB}$, $N=100$ and $\varepsilon_{0}=5 \times 10^{-6}$, then interpolated the resulting values for each and every one of the parameters, i.e., $\eta_{0}, \eta_{1}$ and $\eta_{2}$, in the range of $\sigma_{y} \in[0.01,6)$ for UWB systems and in the range of $\sigma_{y} \in[6,12]$ for other conventional wireless systems. The results are

$$
\left\{\begin{array}{rlr}
\eta_{0} & =\left(-1.0658 \times 10^{-4} \sigma_{y}^{3}-0.0019047 \sigma_{y}^{2}\right. & \\
& \left.+0.083954 \sigma_{y}-0.0004047\right) \sigma_{y} / \sqrt{2}, & \sigma_{y} \in[0.01,6) \mathrm{dB}, \\
& =\left(8.7552 \times 10^{-5} \sigma_{y}^{3}-0.0043629 \sigma_{y}^{2}\right. & \\
& \left.+0.093625 \sigma_{y}-0.011684\right) \sigma_{y} / \sqrt{2}, & \\
\eta_{1} & =-0.0160 \sigma_{y}^{2}+0.3180 \sigma_{y}+1.6580, & \sigma_{y} \in[6,12] \mathrm{dB}, \\
& =-0.0080 \sigma_{y}^{2}+0.2200 \sigma_{y}+1.9580, & \sigma_{y} \in[6,12] \mathrm{dB}, \\
\eta_{2} & =\left(0.045 \sigma_{y}+0.385\right) \ln (10), & \sigma_{y} \in[0.01,12] \mathrm{dB},
\end{array}\right.
$$

such that $\eta_{0} \in\left[3.7 \times 10^{-7}, 5.3869\right], \eta_{1} \in[1.658,3.446]$, and $\eta_{2} \in[0.8865,2.1299]$.

Finally, by adding (10) and (11) together and by using the values of $\eta_{0}, \eta_{1}$ and $\eta_{2}$ provided above, we obtain a tight and generic closed-form approximation of the ergodic capacity as follows, $C_{\mathrm{e}} \simeq$

$\widehat{C}_{\mathrm{e}}=2 \lambda\left[\ln \left(2 \cosh \left(\frac{\widehat{\gamma}_{\mathrm{dB}}}{2 \kappa}\right)\right)+\frac{1}{2 \kappa}\left(\widehat{\gamma}_{\mathrm{dB}}+\frac{\eta_{0}}{\cosh ^{\eta_{1}}\left(\frac{\widehat{\gamma}_{\mathrm{dB}}}{2 \kappa \eta_{2}}\right)}\right)\right]$,

which differs from $C_{\mathrm{e}}$ in (5) by less than $2 \times 10^{-4}$ nats $/ \mathrm{s} / \mathrm{Hz}$ for $\sigma_{y} \in[0.01,6) \mathrm{dB}$ and $8 \times 10^{-3}$ nats $/ \mathrm{s} / \mathrm{Hz}$ for $\sigma_{y} \in[6,12]$ $\mathrm{dB}$, regardless of $\widehat{\gamma}_{\mathrm{dB}}$.

In Figs. 3 and 4, we compare our closed-form approximation $\widehat{C}_{\mathrm{e}}$ in (13) with the closed-form approximation $\widetilde{C}_{\mathrm{e}}=\left[1+2 \exp \left(\frac{\widehat{\gamma}_{\mathrm{dB}}}{\kappa}+\frac{\sigma_{y}^{2}}{2 \kappa^{2}}\right)+\exp \left(2\left(\frac{\widehat{\gamma}_{\mathrm{dB}}}{\kappa}+\frac{\sigma_{y}^{2}}{\kappa^{2}}\right)\right)\right]^{-\frac{1}{2}}$ $\times\left(1+\exp \left(\frac{\widehat{\gamma}_{\mathrm{dB}}}{\kappa}+\frac{\sigma_{y}^{2}}{2 \kappa^{2}}\right)^{2}\right)$, the computable formula $\bar{C}_{\mathrm{e}}=$ $\left\{\sum_{k=1}^{8} a_{k}\left[\operatorname{erfcx}\left(\frac{k \sigma_{y}}{\sqrt{2} \kappa}+\frac{\hat{\gamma}_{\mathrm{dB}}}{\sqrt{2} \sigma_{y}}\right)+\operatorname{erfcx}\left(\frac{k \sigma_{y}}{\sqrt{2} \kappa}-\frac{\hat{\gamma}_{\mathrm{dB}}}{\sqrt{2} \sigma_{y}}\right)\right]\right\}$ $\times 0.5 e^{-\frac{\hat{\gamma}_{\mathrm{dB}}^{2}}{2 \sigma_{y}^{2}}}+\frac{\sigma_{y}}{\sqrt{2 \pi} \kappa} e^{-\frac{\hat{\gamma}_{\mathrm{dB}}^{2}}{2 \sigma_{y}^{2}}}+\frac{\hat{\gamma}_{\mathrm{dB}}}{2 \kappa} \operatorname{erfc}\left(-\frac{\hat{\gamma}_{\mathrm{dB}}}{\sqrt{2} \sigma_{y}}\right)$, which have

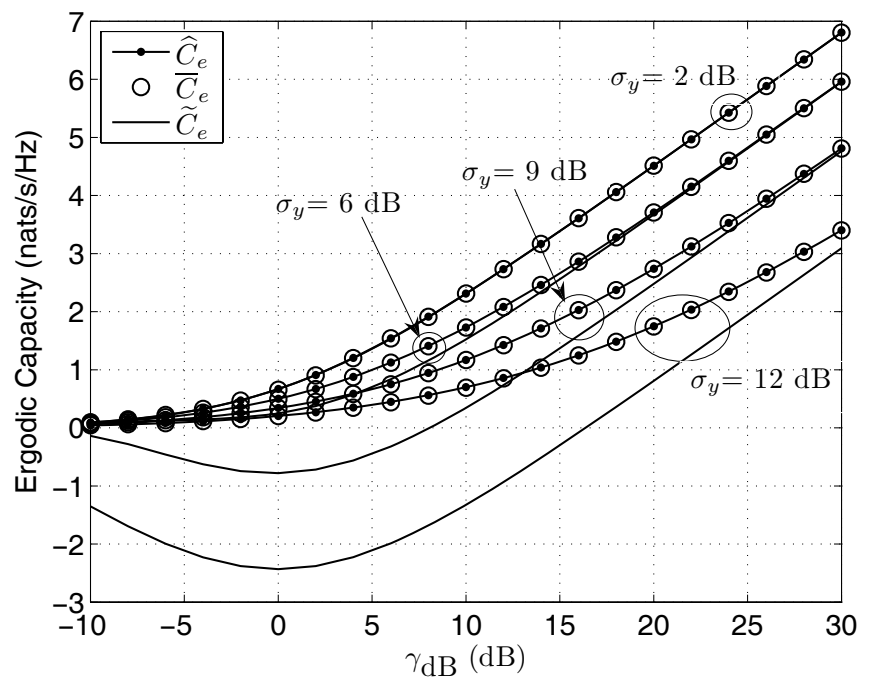

Fig. 4. Comparison of $\widehat{C}_{\mathrm{e}}, \bar{C}_{\mathrm{e}}$, and $\widetilde{C}_{\mathrm{e}}$ vs. $\gamma_{\mathrm{dB}}$, for various $\sigma_{y}$ values, and $m_{y}=-\sigma_{y}^{2} / 2 \kappa$.

both been recently proposed in [14], and $C_{\mathrm{e}}$ in (5) as functions of $\widehat{\gamma}_{\mathrm{dB}}$, for various $\sigma_{y}$ values, and $m_{y}=-\sigma_{y}^{2} / 2 \kappa$. Figure 3 exhibits a highly tight fitness between $C_{\mathrm{e}}$ and $\widehat{C}_{\mathrm{e}}$ for any $\mathrm{dB}$ spread value, hence, it indicates graphically the accuracy of our proposed closed-form approximation in (13). Figure 4 depicts that our proposed closed-form approximation is far more tight than $\widetilde{C}_{\mathrm{e}}$, especially for low SNR values and medium to high $\sigma_{y}$ values. It also shows that our closed-form provides the same degree of accuracy as the formula $\bar{C}_{\mathrm{e}}$, but with a more simplified expression. Notice that the accuracy of our closed-form expression $\widehat{C}_{\mathrm{e}}$ can be improved for lower $\varepsilon_{0}$ values by appropriate choices of the parameters $\eta_{0}, \eta_{1}$ and $\eta_{2}$ in (12).

\section{Applications of $\widehat{C}_{e}$}

\section{A. Capacity of the log-normal fading channel at high SNRs}

Our closed-form expression in (13) can be used to interpret the behavior of the ergodic capacity $C_{\mathrm{e}}$ at high SNRs. Assuming in (13) that $\widehat{\gamma}_{\mathrm{dB}} \gg 2 \kappa \eta_{2}$, it implies that the term $1 / \cosh ^{\eta_{1}}\left(\frac{\hat{\gamma}_{\mathrm{AB}}}{2 \kappa \eta_{2}}\right)$ is equivalent to $e^{-\eta_{1}\left(\frac{\hat{\gamma}_{\mathrm{AB}}}{2 \kappa \eta_{2}}-\ln (2)\right)}$, which goes towards zero as $\widehat{\gamma}_{\mathrm{dB}}$ increases since $\eta_{1}, \eta_{2}$, and $\kappa$ are positive constants. Therefore, $C_{\mathrm{e}} \simeq \widehat{C}_{\mathrm{e}} \simeq C_{\mathrm{e}}^{0}$ in (10) at high SNRs. Moreover, knowing that the term $\ln \left(2 \cosh \left(\frac{\hat{\gamma}_{\mathrm{dB}}}{2 \kappa}\right)\right)$ in (10) is equivalent to $\frac{\widehat{\gamma}_{\mathrm{dB}}}{2 \kappa}$ for $\widehat{\gamma}_{\mathrm{dB}} \gg 2 \kappa$, the ergodic capacity of a log-normal fading channel can be simplified at high SNRs as follows

$$
C_{\mathrm{e}} \simeq \frac{2 \lambda}{\kappa}\left(\gamma_{\mathrm{dB}}+m_{y}\right) .
$$

Thus, the capacity is a linear function of the SNR at high SNRs, which is consistent with the result obtained in [24]. Also, notice that for $m_{y}=0$, this capacity is equal to the capacity of an AWGN channel, i.e., $2 \lambda \ln \left(1+\exp \left(\gamma_{\mathrm{dB}} / \kappa\right)\right)$ [25], at high SNRs. 


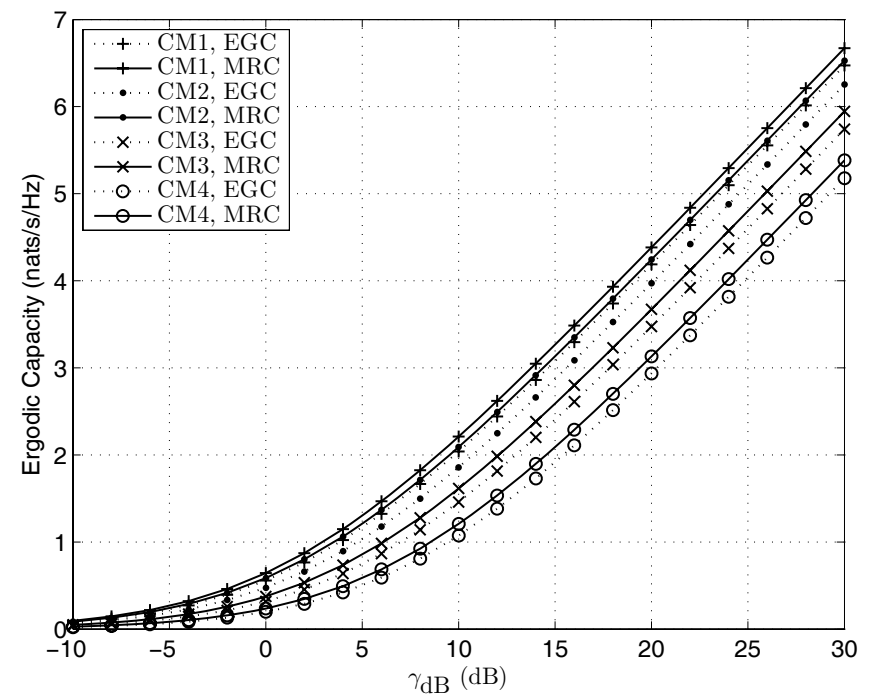

Fig. 5. Ergodic capacity of SISO-UWB systems for different channel scenarios and various combining methods.

\section{B. Capacities of SISO and MIMO-UWB systems over the IEEE 802.15.3a channel model}

Our closed-form expression in (13) can also be used to calculate effectively and accurately the ergodic capacity of communication systems over log-normal fading channels. Here, we provide some results concerning the ergodic capacities of SISO and MIMO-UWB systems operating over the IEEE 802.15.3a channel.

In a MIMO-UWB system, assuming perfect channel estimation and accurate timing synchronization, the receiver output SNR associated with equal gain combining (EGC) and maximum ratio combining (MRC) can be expressed as $\bar{\gamma}=\gamma \alpha$, with $\alpha$ defined as follows [23]

$$
\alpha=\left\{\begin{array}{cc}
\left(\sum_{\rho=0}^{N_{\mathrm{r}}-1} \sum_{n=0}^{N_{\mathrm{t}}-1} \sum_{f=0}^{N_{\mathrm{f}}-1}\left|\beta_{\rho, n, f}\right|\right)^{2} /\left(N_{\mathrm{r}} N_{\mathrm{t}}^{2} N_{\mathrm{f}}\right) & \text { for EGC, } \\
\sum_{\rho=0}^{N_{\mathrm{r}}-1} \sum_{n=0}^{N_{\mathrm{t}}-1} \sum_{f=0}^{N_{\mathrm{f}}-1} \beta_{\rho, n, f}^{2} / N_{\mathrm{t}} & , \text { for MRC, }
\end{array}\right.
$$

respectively, where $N_{\mathrm{r}}$ is the number of receive antennas, $N_{\mathrm{t}}$ is the number of transmit antennas, and $N_{\mathrm{f}}$ is the number of multipath over each receive-transmit antenna pairs.

As explained in [19]-[22], the sum of independent or correlated log-normal RVs can be well-approximated by an equivalent log-normal RV. Thus, the effect of the log-normal fading channel upon any communication system can be modeled by using an equivalent log-normal RV. For instance, it has been shown in [23] that the effect of several independent log-normal multipath attenuation factors of the IEEE 802.15.3a channel model can be appropriately combined into an equivalent lognormal RV. Numerical values of the mean $m_{\alpha}$ and standard deviation $\sigma_{\alpha}$ of the equivalent log-normal RV $\alpha$ have been obtained considering either MRC or EGC, various numbers of multipath, and different numbers of transmit antennas, in Tables 4.2, 4.3, 5.1 and 5.2 of [23]. These values have been first derived by using a theoretical approach and then verified

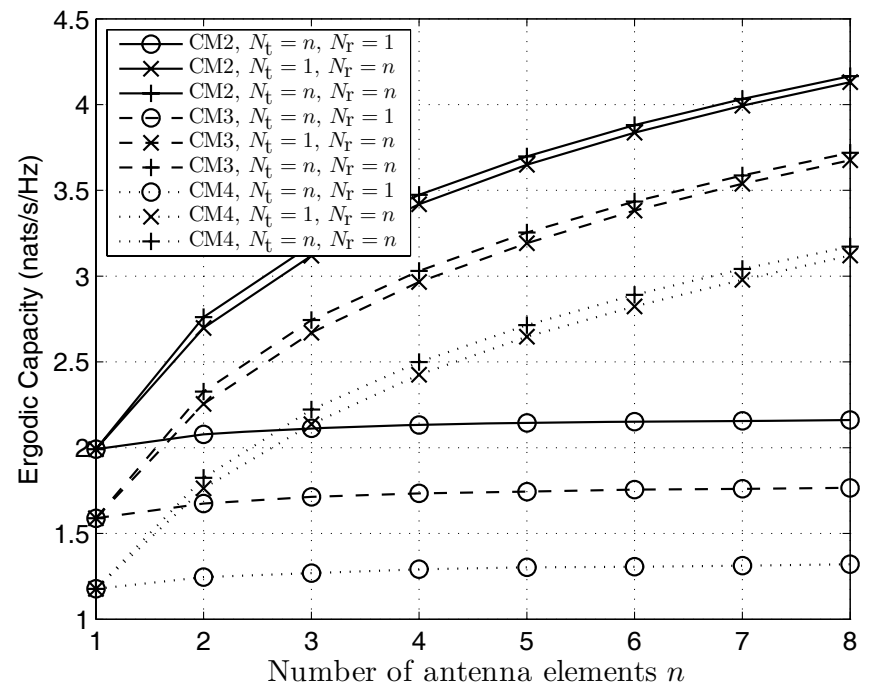

Fig. 6. Ergodic capacity of MIMO-UWB systems for different channel scenarios and various numbers of transmit and receive antennas.

through simulations.

Inserting the values of $m_{\alpha}$ and $\sigma_{\alpha}$ from the Tables 4.2, 4.3 of [23] in (13), the ergodic capacity of a SISO-UWB system over various channel scenarios of the IEEE 802.15.3a channel model, i.e., CM1-4 [2], is plotted in Fig. 5, by applying either EGC or MRC, for $N_{\mathrm{f}}=20, N_{\mathrm{t}}=1$, and $N_{\mathrm{r}}=1$. The results show that MRC provides an extra 0.2 nats $/ \mathrm{s} / \mathrm{Hz}$ capacity improvement compared to EGC, and the capacity over the line-of-sight (LOS) channel CM1 is 1.2 nats $/ \mathrm{s} / \mathrm{Hz}$ higher than that over the worst non-LOS channel scenario CM4. Notice also that all the curves tend to be parallel at high SNRs, which is consistent with the result in (14). Therefore, the ergodic capacity difference between any two systems $A$ and $B$ operating over a log-normal fading channel is constant at high SNRs and can be given by

$$
\Delta C_{\mathrm{e}}^{A, B}=2 \lambda\left|m_{\alpha}^{A}-m_{\alpha}^{B}\right|,
$$

where $m_{\alpha}^{A}$ and $m_{\alpha}^{B}$ are the mean values of the RVs $\alpha_{A}$ and $\alpha_{B}$ for systems $A$ and $B$, respectively.

In Fig. 6, the ergodic capacity of a MIMO-UWB system over various channel scenarios, i.e., CM2-4, is plotted against the number of antenna elements $n$ which is considered at the transmitter or at the receiver, by applying MRC with $N_{\mathrm{f}}=20$, and for $\gamma_{\mathrm{dB}}=10 \mathrm{~dB}$. Using (15) and the same method as in [23], we have obtained values of $m_{\alpha}$ and $\sigma_{\alpha}$ for the different antenna configurations considered in Fig. 6 beforehand. The results show that if the number of receive antennas is equal to one, $N_{\mathrm{r}}=1$, increasing the number of transmit antennas has a very limited effect on the capacity, which then saturates very fast. On the other hand, if the number of transmit antennas is equal to one, $N_{\mathrm{t}}=1$, the capacity increases in a logarithm way with the number of receive antennas. These results are in line with those obtained for a MIMO Rayleigh-fading channel [25]. However, if the number of transmit antennas is equal to the number of receive antennas the capacity still increases in a logarithm way with the number of receive antennas. This result is very different from the MIMO Rayleigh-fading channel [25], where it is well-known that the capacity increases lin- 
early with the number of transmit/receive antennas, i.e., when $n=N_{\mathrm{t}}=N_{\mathrm{r}}$. As it has been indicated in [23], increasing the number of transmit antennas over the IEEE 802.15.3a channel has a limited impact on the performance improvement in terms of bit error probability. A SNR gain of about 3 to $4 \mathrm{~dB}$ can be observed when using one received antenna and two instead of one transmit antenna over CM2. This gain is marginal compared to the $25 \mathrm{~dB}$ gain obtained in the MIMO Rayleighfading case. Thus, it can be concluded that mainly receive diversity can be used to increase the capacity of MIMO-UWB systems operating over the IEEE 802.15.3a channel.

\section{CONCLUSION}

A tight closed-form approximation of the ergodic capacity for wireless communication systems operating over lognormal fading channels has been derived. Its accuracy has been maintained by finding values of parameters $\eta_{0}, \eta_{1}$ and $\eta_{2}$ that minimize a MSE criterion, and has been shown experimentally for practical SNR and $\mathrm{dB}$ spread values. Our expression has been used to interpret the behavior of the ergodic capacity of log-normal fading channels at high SNRs, as well as to provide a simple criterion for comparing the capacities of systems operating over log-normal fading channels. Finally, its application in evaluating the ergodic capacities of SISO and MIMO-UWB systems over the IEEE 802.15.3a channel model has been presented, and it has been shown that multi-antennas UWB systems can mainly benefit from receive diversity to increase their capacities over the IEEE 802.15.3a channel.

\section{REFERENCES}

[1] J. Proakis, Digital Communications, 4th ed.. McGraw-Hill, 2001.

[2] J. Foerster and et al., "Channel modelling sub-committee report final," IEEE P802.15 Wireless Personal Area Networks, Tech. Rep. P802.1502/490r1-SG3a, Feb. 2003.

[3] M. Ghavami, L. Michael, and R. Kohno, Ultra Wideband Signals and Systems in Communication Engineering. Wiley Europe, 2004.

[4] A. Laourine, A. Stephenne, and S. Affes, "Capacity of log-normal fading channels," in Proc. IWCMC '07, Honolulu, Hawaii, Aug. 2007, pp. 1317.

[5] W. C. Y. Lee, "Estimate of channel capacity in Rayleigh fading environment," IEEE Trans. Veh. Technol., vol. 93, no. 3, pp. 187-189, Aug. 1990.

[6] S. Khatalin and J. P. Fonseka, "On the channel capacity in Rician and Hoyt fading environment with MRC diversity," IEEE Trans. Veh. Technol., vol. 55, no. 1, pp. 137-141, Jan. 2006.

[7] N. C. Sagias, G. S. Tombras, and G. K. Karagiannidis, "New results for the Shannon channel capacity in generalized fading channels," IEEE Commun. Lett., vol. 9, no. 2, pp. 97-99, Jan. 2005.
[8] M. K. Simon and M.-S. Alouini, Digital Communication over Fading Channels: A Unified Approach to Performance Analysis. Wiley, 2000.

[9] D. B. da Costa and M. D. Yacoub, "Average channel capacity for generalized fading scenarios," IEEE Commun. Lett., vol. 11, no. 12, pp. 949-951, Dec. 2007.

[10] G. Fraidenraich, O. Leveque, and J. M. Cioffi, "On the MIMO channel capacity for the dual and asymptotic cases over Hoyt channels," IEEE Commun. Lett., vol. 11, no. 1, pp. 31-33, Jan. 2007.

[11] _ - "On the MIMO channel capacity for the Nakagami- $m$ channel," IEEE Trans. Inform. Theory, vol. 54, no. 8, pp. 3752-3757, Aug. 2008.

[12] F. Héliot, M. Ghavami, and M. R. Nakhai, "An accurate closed-form approximation of the average probability of error over a log-normal fading channel," IEEE Trans. Wireless Commun., vol. 7, no. 5, pp. 14951500, May 2008.

[13] M. S. Alouini and A. J. Goldsmith, "Area spectral efficiency of cellular mobile radio systems," IEEE Trans. Veh. Technol., vol. 48, no. 4, pp. 1047-1066, July 1999.

[14] A. Laourine, A. Stephenne, and S. Affes, "Estimating the ergodic capacity of log-normal channels," IEEE Commun. Lett., vol. 11, no. 7, pp. 568-570, July 2007.

[15] N. C. Beaulieu and F. Rajwani, "Highly accurate simple closed-form approximations to lognormal sum distributions and densities," IEEE Commun. Lett., vol. 8, no. 12, pp. 709-711, Dec. 2004.

[16] F. Héliot, X. Chu, R. Hoshyar, and R. Tafazolli, "An accurate closedform approximation of the ergodic capacity over log-normal fading channels," in Proc. IEEE PIMRC 08, Cannes, France, Sept. 2008.

[17] J. Gubner and K. Hao, "An exact computable formula for the average bit-error probability of the IEEE 802.15.3a UWB channel model," in Proc. IEEE ICUW 2005, Zurich, Switzerland, Sept. 2005, pp. 142-146.

[18] H. Liu, "Error performance of a pulse amplitude and position modulated ultra-wideband system over lognormal fading channel," IEEE Commun. Lett., vol. 7, no. 11, pp. 531-533, Nov. 2003.

[19] N. C. Beaulieu, A. A. Abu-Dayya, and P. J. Mclane, "Estimating the distribution of a sum of independent lognormal random variables," IEEE Trans. Commun., vol. 43, no. 12, pp. 2869-2873, Dec. 1995.

[20] J. S. Filho, P. Cardieri, and M. Yacoub, "Simple accurate lognormal approximation to lognormal sums," Electron. Lett., vol. 41, no. 18, pp. 1016-1017, Sept. 2005.

[21] _ , "Highly accurate range-adaptive lognormal approximation to lognormal sum distributions," Electron. Lett., vol. 42, no. 6, pp. 361$363,2006$.

[22] N. B. Mehta, A. F. Molisch, J. Wu, and J. Zhang, "Approximating a sum of random variables with a lognormal," IEEE Trans. Wireless Commun., vol. 6, no. 7, pp. 2690-2699, July 2007.

[23] F. Héliot, "Design and analysis of space-time block and trellis coding schemes for single-band UWB communications systems," Ph.D. dissertation, King's College London, University of London, July 2006. [Online]. Available: http://membres.lycos.fr/fheliot/pub/PhD_thesis.pdf

[24] M. Saleem, B. T. Sieskul, and T. Kaiser, "Channel capacity assessments in UWB communication system over lognormal fading," in Proc. IET Seminar on UWB Systems, Technologies and Applications, Apr. 2006, pp. $155-159$.

[25] M. Dohler, "Virtual antenna arrays," Ph.D. dissertation, King's College London, University of London, Nov 2003. [Online]. Available: http://perso.rd.francetelecom.fr/dohler/theses/PhD.pdf 